OCU-PHYS 404

AP-GR 111

\title{
Comparison of two approximation schemes for solving perturbations in a LTB cosmological model
}

\author{
${ }^{1}$ Ryusuke Nishikawa *, ${ }^{1}$ Ken-ichi Nakao ${ }^{\dagger}$, and ${ }^{2}$ Chul-Moon Yoo ${ }^{\ddagger}$, \\ ${ }^{1}$ Department of Mathematics and Physics, \\ Graduate School of Science, Osaka City University, \\ 3-3-138 Sugimoto, Sumiyoshi, Osaka 558-8585, Japan \\ ${ }^{2}$ Division of Particle and Astrophysical Science, \\ Graduate School of Science, Nagoya University, \\ Furo-cho, Chikusa-ku, Nagoya 464-8602, Japan
}

\begin{abstract}
Recently, the present authors studied perturbations in the Lemaître-Tolman-Bondi cosmological model by applying the second-order perturbation theory in the dust Friedmann-LemaitreRobertson-Walker universe model. Before this work, the same subject was studied in some papers by analyzing linear perturbations in the Lemaitre-Tolman-Bondi cosmological model under the assumption proposed by Clarkson, Clifton and February, in which two of perturbation variables are negligible. However, it is a non-trivial issue in what situation the Clarkson-Clifton-February assumption is valid. In this paper, we investigate differences between these two approaches. It is shown that, in general, these two approaches are not compatible with each other. That is, in our perturbative procedure, the Clarkson-Clifton-February assumption is not valid at the order of our interest.
\end{abstract}

\footnotetext{
* E-mail:ryusuke@sci.osaka-cu.ac.jp

$\dagger$ E-mail:knakao@sci.osaka-cu.ac.jp

$\ddagger$ E-mail:yoo@gravity.phys.nagoya-u.ac.jp
} 


\section{INTRODUCTION}

Most of modern cosmological models are based on the Copernican principle which states we are not living at a privileged position in the universe. The observed isotropy of the Cosmic Microwave Background (CMB) radiation together with the Copernican principle implies our universe is homogeneous and isotropic, if the small scale structures less than $50 \mathrm{Mpc}$ are coarse-grained. Although the standard cosmology can explain a lot of observations naturally, it should be noted that the Copernican principle on cosmological scales $\geq 1 \mathrm{Gpc}$ has not been confirmed. This means modern cosmology would contain systematic errors that arise from non-Copernican inhomogeneities of the background universe, which is usually unexpected. The systematic errors may mislead us when we consider major issues in modern cosmology such as testing general relativity at cosmological scales and probing dark energy abundance. Thus, it is an essential task in modern precision cosmology to test the Copernican principle.

In order to test the Copernican principle, we have to investigate non-Copernican cosmological models which drop the Copernican principle. Non-Copernican models commonly assume that we live close to the center in a spherically symmetric spacetime since the universe is observed to be nearly isotropic around us. These models have also been studied as an alternative to dark energy, because some of them can explain the observational data of the luminosity distances of Type Ia supernovae without introducing dark energy [1 14$]$. The non-Copernican models without dark energy have been tested by observations including the CMB acoustic peaks [15 26], the present Hubble parameter $H_{0}[17,18,20,21,23]$, the Baryon Acoustic Oscillation scale of the galaxy correlations [17, 27, 28], the kinematic

Sunyaev-Zeldovich effect [29 34] and others [35 56], and significant observational constraints exist, although they have never been completely excluded yet. However, it should be noted that even if we accept dark energy components, the existence of the large spherical inhomogeneity may significantly affects observational results (see e.g. Ref. [57, 58]). A large void universe model which assumes we live at a center of a huge spherical void whose radius is larger than $1 \mathrm{Gpc}$ is known as one of popular models among the non-Copernican cosmologies, and we take such model into consideration in this paper.

Growth of the large-scale structure in the universe can be thought of as one of the most useful tools to investigate the large spherical void model, because the evolution of perturbations is expected to reflect the tidal force in the background spacetime. Unfortunately, linear perturbation equations in the spherical void universe cannot be solved analytically in general situation [59], because the number of isometries in a spherically symmetric inhomogeneous spacetime is too little.

Recently, linear perturbations in the LTB cosmological model and the observations related to them have been studied by several researchers [43, 60 65]: Zibin [65] and Dunsby, Goheer, Osano and Uzan [43] solved perturbations by using "silent universe approximation" in which the magnetic part of the Weyl tensor vanishes. However, we should note that the magnetic part of the Weyl tensor usually plays an important role even in Newtonian situations [66], and hence the silent universe model may fail to include the situations of our interest. Clarkson Clifton and February [62] (hereafter CCF) classified perturbations into the "scalar", "vector" 
and "tensor" degrees of freedom by their behaviors in the limit to the homogeneous and isotropic background, and solved perturbation equations by assuming that the "vector" and "tensor" modes are negligible. However, in the case of the LTB spacetime, the vector and tensor modes couple to the scalar mode in general. It is not clear whether CCF's assumption is valid in the situations of our interest. In our previous works [67, 68], we studied the same subject by analyzing perturbations in the Einstein-de Sitter(EdS) universe model up to the second order without any additional assumptions and revealed the growth rate of the density perturbations and their two point correlation functions. In this paper, we study the validity of CCF's assumption within the non-linear perturbation approach that we have adopted in our previous works.

We adopt the same sign conventions of the metric and the Riemann tensors as those in Ref. [69].

\section{A REVIEW ON PREVIOUS WORK BY CLARKSON-CLIFTON-FEBRUARY}

First, we review linear perturbations in the LTB spacetime. The infinitesimal world interval and the stress-energy tensor are given by

$$
\begin{aligned}
& d s^{2}=-d t^{2}+\frac{a_{\|}^{2}(t, r)}{1-k(r) r^{2}} d r^{2}+a_{\perp}^{2}(t, r) r^{2} d \Omega^{2}, \\
& T_{\mu \nu}=\rho^{\mathrm{LTB}}(t, r) \bar{u}_{\mu} \bar{u}_{\nu},
\end{aligned}
$$

where $a_{\|}=\partial_{r}\left(r a_{\perp}\right)$ and $\bar{u}_{\mu}=(-1,0,0,0)$. Because of the spherical symmetry of the LTB spacetime, perturbations in the LTB spacetime can be decoupled into two independent modes, called the polar and the axial modes (for details, see [62]). Since we are interested in the evolution of the density perturbations, we focus on the polar mode. The metric of the perturbed LTB spacetime in the Regge-Wheeler (RW) gauge is written as

$$
\begin{aligned}
d s^{2} & =-[1+(2 \tilde{\eta}-\tilde{\chi}-\tilde{\varphi})] d t^{2}-2 \frac{\tilde{\varsigma} a_{\|}}{\sqrt{1-k r^{2}}} d t d r \\
& +[1+(\tilde{\chi}+\tilde{\varphi})] \frac{a_{\|}^{2}}{1-k r^{2}} d r^{2}+a_{\perp}^{2} r^{2}(1+\tilde{\varphi}) d \Omega^{2}
\end{aligned}
$$

where $\tilde{\eta}(t, \mathbf{x}), \tilde{\chi}(t, \mathbf{x}), \tilde{\varphi}(t, \mathbf{x})$ and $\tilde{\varsigma}(t, \mathbf{x})$ are polar perturbations, and their expansion coefficients of a spherical harmonic expansion correspond to $\eta(t, r), \chi(t, r), \varphi(t, r)$ and $\varsigma(t, r)$ in CCF's paper, respectively [62]. The density and 4-velocity of the perturbed spacetime are given by

$$
\begin{aligned}
\rho & =\rho^{\operatorname{LTB}}(1+\tilde{\delta}) \\
u_{\mu} & =\left(-1-\frac{1}{2}(2 \tilde{\eta}-\tilde{\chi}-\tilde{\varphi}), \frac{a_{\|}(\tilde{w}-\tilde{\varsigma} / 2)}{\sqrt{1-k r^{2}}}, \partial_{\theta} \tilde{v}, \partial_{\varphi} \tilde{v}\right),
\end{aligned}
$$

where $\tilde{\delta}(t, \mathbf{x}), \tilde{w}(t, \mathbf{x})$ and $\tilde{v}(t, \mathbf{x})$ are polar perturbations, and their expansion coefficients of a spherical harmonic expansion correspond to $\Delta(t, r), w(t, r)$ and $v(t, r)$ in CCF's paper, 
respectively. By substituting the expressions (3), (4) and (5) into the Einstein equations, we obtain the perturbation equations (see Eqs. (3.1)-(3.7) in CCF). From one of the perturbation equations, we obtain $\tilde{\eta}=0$ in general (see Eq. (3.4) in CCF). However, other perturbation equations are complicated and cannot be reduced to ordinary differential equations differently from that in the homogeneous and isotropic universe models.

In section 3 of Ref. [62], CCF analyzed the equation for the density perturbation by assuming

$$
\tilde{\chi}=\tilde{\varsigma}=0 .
$$

By virtue of this assumption, the perturbation equations are reduced to very simple forms (see also [63]), and hence CCF succeeded in obtaining the analytic solutions of the equations for the perturbations, $\tilde{\varphi}$ and $\tilde{\delta}$. However, the range of the validity of this assumption is not clear in contrast to our perturbative procedure. We check the validity within our perturbative procedure in the next section.

\section{PERTURBATIVE ANALYSIS OF CCF PROCEDURE}

In our previous works [67, 68], we studied perturbations in the LTB universe model by applying the second-order perturbation theory for the dust-filled FLRW universe model. We introduced perturbations parametrized by two small expansion parameters $\kappa$ and $\epsilon$. The limit $\epsilon \rightarrow 0$ leads to the exact LTB solution, if we take all orders of $\kappa$ into account. By contrast, the limit $\kappa \rightarrow 0$ with $0<\epsilon \ll 1$ leads to the homogeneous and isotropic universe with small anisotropic perturbations. Then, in order to see the effect of the non-Copernican structure on the evolution of the anisotropic perturbations, we studied the non-linear effects up to the order of $\kappa \epsilon$. Here, it should be noted that we included only the scalar mode for perturbations of the order $\epsilon$, since the vector and the tensor modes of the linear perturbations in the homogeneous and isotropic universes do not grow with time as is well known. We studied perturbations in the synchronous comoving gauge, while CCF studied in the RW gauge. To compare the two approaches, we derive perturbation equations of our approach in the RW gauge.

In order to apply our method, we regard the background LTB spacetime in Eqs. (11) and (2) as isotropic perturbations in the EdS universe model which are characterized by $\kappa$ which should be regarded as a book-keeping parameter here: it is replaced by unity after deriving the perturbation equations. By introducing isotropic perturbations $\ell_{\|}^{(1)}(t, r), \ell_{\perp}^{(1)}(t, r)$ and $\Delta^{(1)}(t, r)$ (for details, see [67]), we write the functions $a_{\|}, a_{\perp}, k(r)$ and $\rho^{\mathrm{LTB}}$ in the form

$$
\begin{aligned}
a_{\|} & =a\left[1+\frac{\kappa}{2} \partial_{r}\left(r \ell_{\perp}^{(1)}\right)\right]+\mathcal{O}\left(\kappa^{2}\right), \\
a_{\perp} & =a\left[1+\frac{\kappa}{2} \ell_{\perp}^{(1)}\right]+\mathcal{O}\left(\kappa^{2}\right), \\
k(r) r^{2} & =\kappa\left[\ell_{\|}^{(1)}-\partial_{r}\left(r \ell_{\perp}^{(1)}\right)\right]+\mathcal{O}\left(\kappa^{2}\right), \\
\rho^{\mathrm{LTB}} & =\bar{\rho}\left[1+\kappa \Delta^{(1)}\right]+\mathcal{O}\left(\kappa^{2}\right),
\end{aligned}
$$


where $a$ and $\bar{\rho}$ are the scale factor and the matter density in the background EdS universe. In accordance with the prescription in our previous paper [67], we write the polar modes of perturbations as follows:

$$
\tilde{\varphi}=\epsilon \tilde{\varphi}^{(1)}+\kappa \epsilon \tilde{\varphi}^{(2)}+\mathcal{O}\left(\kappa^{2} \epsilon\right)
$$

and $\tilde{\chi}, \tilde{\varsigma}, \tilde{\delta}, \tilde{w}$ and $\tilde{v}$ are written in the similar forms. Assuming $\epsilon \ll \kappa<1$ and the Einstein equations hold in each order with respect to $\kappa$ and $\epsilon$, we can obtain the equations for the perturbations of the order $\epsilon$ and $\kappa \epsilon$.

First of all, we see the perturbation equations of the order $\epsilon$. The perturbations of the order $\epsilon$ are equivalent to those of the EdS universe model. As mentioned above, we only include the scalar mode of the order $\epsilon$ in our present analysis. By contrast, the polar perturbations may be a mixture of the scalar, vector and tensor modes in the context of the perturbation theory for the EdS universe model. Thus, we need to relate the polar perturbations to the scalar, vector and tensor perturbations in the EdS universe model. CCF derived these relations in Eqs. (4.5)-(4.8) in their paper, by taking the homogeneous limit of LTB universe model. From Eqs. (4.6) and (4.7) in CCF, we can see that $\tilde{\varsigma}^{(1)}$ and $\tilde{\chi}^{(1)}$ do not contain the scalar mode but the vector and tensor modes. Thus, the neglect of the vector and tensor modes leads to

$$
\tilde{\varsigma}^{(1)}=\tilde{\chi}^{(1)}=0 .
$$

In the case that Eq. (12) holds, up to the order of $\epsilon$, the perturbed metric (3) becomes

$$
d s^{2}=-\left(1-\tilde{\varphi}^{(1)}\right) d t^{2}+a^{2}\left(1+\tilde{\varphi}^{(1)}\right)\left(d r^{2}+r^{2} d \Omega^{2}\right) .
$$

It is clear from the above equation that the metric perturbation of the order $\epsilon$ is the scalar mode. Correspondingly, the perturbations of $\rho$ and $u^{\mu}$ of the order $\epsilon$ contain the only scalar mode.

By substituting the expressions (3), (44) and (5) into the Einstein equations and using the expansions (17)-(11) together with Eq. (12), we obtain the perturbation equations of the order $\epsilon$ as

$$
\begin{aligned}
\ddot{\tilde{\varphi}}^{(1)}+4 H \dot{\tilde{\varphi}}^{(1)} & =0, \\
8 \pi \bar{\rho} \tilde{\delta}^{(1)} & =3 H \dot{\tilde{\varphi}}(1)+3 H^{2} \tilde{\varphi}^{(1)}-\frac{1}{a^{2}} \mathcal{D}^{i} \mathcal{D}_{i} \tilde{\varphi}^{(1)}, \\
8 \pi \bar{\rho} \tilde{w}^{(1)} & =\frac{\partial_{r}}{a}\left(\dot{\tilde{\varphi}}^{(1)}+H \tilde{\varphi}^{(1)}\right), \\
8 \pi \bar{\rho} \tilde{v}^{(1)} & =\dot{\dot{\varphi}}(1)+H \tilde{\varphi}^{(1)},
\end{aligned}
$$

where we defined the operator $\mathcal{D}^{i} \mathcal{D}_{i}$ as

$$
\mathcal{D}^{i} \mathcal{D}_{i}:=\frac{1}{r^{2}} \frac{\partial}{\partial r}\left(r^{2} \frac{\partial}{\partial r}\right)+\frac{1}{r^{2} \sin \theta} \frac{\partial}{\partial \theta}\left(\sin \theta \frac{\partial}{\partial \theta}\right)+\frac{1}{r^{2} \sin ^{2} \theta} \frac{\partial^{2}}{\partial^{2} \phi} .
$$

Although it is not clear from only Eq. (5) whether the perturbations of $u_{\mu}$ of the order $\epsilon$ are the scalar mode or the mixture with the vector mode, it is clear from Eqs. (16) and (17) 
that it is purely a scalar mode. Thus, the assumption (6) up to the order $\epsilon$ is equivalent to neglecting the vector and tensor modes.

Next, we see the perturbation equations of the order $\kappa \epsilon$. Substituting the expressions (3) (5) into the Einstein equations and using the expansions (7)-(11), we obtain the perturbation equations of the order $\kappa \epsilon$ as

$$
\begin{aligned}
& -\ddot{\tilde{\chi}}^{(2)}-3 H \dot{\tilde{\chi}}^{(2)}+\frac{1}{a^{2}}\left[\mathcal{D}^{i} \mathcal{D}_{i}-2\left(\frac{2}{r} \partial_{r}-\frac{1}{r^{2}}\right)\right] \tilde{\chi}^{(2)}=2\left(\dot{\ell}_{\|}^{(1)}-\dot{\ell}_{\perp}^{(1)}\right) \dot{\tilde{\varphi}}^{(1)} \\
& +2\left[\left(\ddot{\ell}_{\|}^{(1)}-\ddot{\ell}_{\perp}^{(1)}\right)+3 H\left(\dot{\ell}_{\|}^{(1)}-\dot{\ell}_{\perp}^{(1)}\right)\right] \tilde{\varphi}^{(1)} \\
& \ddot{\tilde{\varphi}}^{(2)}+4 H \dot{\tilde{\varphi}}^{(2)}+H \dot{\tilde{\chi}}^{(2)}+\frac{1}{2 a^{2}}\left[\mathcal{D}^{i} \mathcal{D}_{i}-\partial_{r}^{2}-2\left(\frac{2}{r} \partial_{r}-\frac{1}{r^{2}}\right)\right] \tilde{\chi}^{(2)}=-2 \dot{\ell}_{\perp}^{(1)} \dot{\tilde{\varphi}}^{(1)} \\
& +\frac{2}{a^{2} r^{2}}\left(\ell_{\|}^{(1)}-\partial_{r}\left(r \ell_{\perp}^{(1)}\right)\right) \tilde{\varphi}^{(1)} \\
& \dot{\tilde{\zeta}}^{(2)}+2 H \tilde{\varsigma}^{(2)}+\frac{\partial_{r} \tilde{\chi}^{(2)}}{a}=0
\end{aligned}
$$

$$
\begin{aligned}
8 \pi \bar{\rho} \tilde{\delta}^{(2)} & =3 H \dot{\tilde{\varphi}}^{(2)}+3 H^{2} \tilde{\varphi}^{(2)}-\frac{1}{a^{2}} \mathcal{D}^{i} \mathcal{D}_{i} \tilde{\varphi}^{(2)} \\
& +H \dot{\tilde{\chi}}^{(2)}+\left[3 H^{2}+\frac{1}{2 a^{2}}\left(\partial_{r}^{2}+\frac{4}{r} \partial_{r}+\frac{2}{r^{2}}-\mathcal{D}^{i} \mathcal{D}_{i}\right)\right] \tilde{\chi}^{(2)}+\frac{2 H}{a}\left[\partial_{r}+\frac{2}{r}\right] \tilde{\varsigma}^{(2)} \\
& +\frac{1}{a^{2}}\left(\ell_{\|}^{(1)}-\ell_{\perp}^{(1)}\right) \partial_{r}^{2} \tilde{\varphi}^{(1)}+\frac{1}{a^{2}}\left[\frac{2}{r}\left(\ell_{\|}^{(1)}-\ell_{\perp}^{(1)}\right)-\frac{1}{2} \partial_{r}\left(\ell_{\|}^{(1)}-2 \ell_{\perp}^{(1)}\right)\right] \partial_{r} \tilde{\varphi}^{(1)} \\
& +\frac{1}{a^{2}} \ell_{\perp}^{(1)} \mathcal{D}^{i} \mathcal{D}_{i} \tilde{\varphi}^{(1)}+\frac{1}{2}\left(\dot{\ell}_{\|}^{(1)}+2 \dot{\ell}_{\perp}^{(1)}\right) \dot{\tilde{\varphi}}^{(1)} \\
& +\left[2 H\left(\dot{\ell}_{\|}^{(1)}+2 \dot{\ell}_{\perp}^{(1)}\right)-8 \pi \bar{\rho} \Delta^{(1)}\right] \tilde{\varphi}^{(1)}-8 \pi \bar{\rho} \Delta^{(1)} \tilde{\delta}^{(1)},
\end{aligned}
$$$$
8 \pi \bar{\rho} \tilde{w}^{(2)}=\frac{\partial_{r}}{a}\left(\dot{\tilde{\varphi}}^{(2)}+H \tilde{\varphi}^{(2)}\right)-\frac{1}{a r} \dot{\tilde{\chi}}^{(2)}+\frac{H}{a} \partial_{r} \tilde{\chi}^{(2)}+\frac{1}{2 a^{2}}\left[\partial_{r}^{2}+\frac{2}{r} \partial_{r}-\mathcal{D}^{i} \mathcal{D}_{i}\right] \tilde{\varsigma}^{(2)}
$$$$
+\frac{3}{2} H^{2} \tilde{\varsigma}^{(2)}-\frac{1}{2 a} \ell_{\|}^{(1)} \partial_{r} \dot{\tilde{\varphi}}^{(1)}-\frac{1}{2 a}\left[H \ell_{\|}^{(1)}-\left(2 \dot{\ell}_{\perp}^{(1)}-\dot{\ell}_{\|}^{(1)}\right)\right] \partial_{r} \tilde{\varphi}^{(1)}
$$$$
-8 \pi \bar{\rho} \Delta^{(1)} \tilde{w}^{(1)}
$$

$$
8 \pi \bar{\rho} \tilde{v}^{(2)}=\dot{\tilde{\varphi}}(2)+H \tilde{\varphi}^{(2)}+\frac{1}{2} \dot{\tilde{\chi}}^{(2)}+H \tilde{\chi}^{(2)}+\frac{1}{2 a} \partial_{r} \tilde{\varsigma}^{(2)}+\frac{1}{2} \dot{\ell}_{\|}^{(1)} \tilde{\varphi}^{(1)}-8 \pi \bar{\rho} \Delta^{(1)} \tilde{v}^{(1)}
$$

By introducing the new variable $\tilde{y}$ defined as

$$
\tilde{y}:=\frac{\tilde{\chi}^{(2)}}{r^{2}}
$$


and defining its Fourier transformation $\tilde{y}_{\mathbf{k}}$ as

$$
\tilde{y}(t, \mathbf{x})=\int \frac{d^{3} k}{(2 \pi)^{3 / 2}} e^{i \mathbf{k} \cdot \mathbf{x}} \tilde{y}_{\mathbf{k}}(t)
$$

equation (19) is reduced to

$$
\ddot{\tilde{y}}_{\mathbf{k}}+3 H \dot{\tilde{y}}_{\mathbf{k}}+\frac{k^{2}}{a^{2}} \tilde{y}_{\mathbf{k}}=S_{\mathbf{k}}(t)
$$

where the source term $S_{\mathbf{k}}(t)$ is defined as

$$
\begin{aligned}
S_{\mathbf{k}}(t):= & -\int \frac{d^{3} x}{(2 \pi)^{3 / 2}} e^{-i \mathbf{k} \cdot \mathbf{x}}\left\{2\left(\dot{\ell}_{\|}^{(1)}-\dot{\ell}_{\perp}^{(1)}\right) \frac{\dot{\tilde{\varphi}}^{(1)}}{r^{2}}+2\left[\left(\ddot{\ell}_{\|}^{(1)}-\ddot{\ell}_{\perp}^{(1)}\right)\right.\right. \\
& \left.\left.+3 H\left(\dot{\ell}_{\|}^{(1)}-\dot{\ell}_{\perp}^{(1)}\right)\right] \frac{\tilde{\varphi}^{(1)}}{r^{2}}\right\} .
\end{aligned}
$$

We find from Eqs. (6)-(8) in Ref. 67] that $\ell_{\|}^{(1)}$ and $\ell_{\perp}^{(1)}$ are proportional to the scale factor $a$, and the growing solution of Eq. (14) is $\tilde{\varphi}^{(1)}=$ constant. Hence, the source term $S_{\mathbf{k}}(t)$ is proportional to $a^{-2}$. From this fact, we can see that the particular solution of Eq. (27) is $S_{\mathbf{k}}\left(t_{0}\right) / k^{2}$, where we set $a\left(t_{0}\right)=1$ by using the freedom of the constant re-scaling in the spatial coordinates. By using the fact that the Hubble function and the scale factor are given as $H=2 /(3 t)$ and $a=\left(t / t_{0}\right)^{2 / 3}$ in the EdS universe model, we obtain the homogeneous solutions of Eq. (27) as

$$
\tilde{y}_{\mathbf{k}, 1}(t)=\frac{1}{k t_{0}^{2 / 3} t^{1 / 3}} j_{1}\left(3 k t_{0}^{2 / 3} t^{1 / 3}\right), \quad \text { and } \quad \tilde{y}_{\mathbf{k}, 2}(t)=\frac{1}{k t_{0}^{2 / 3} t^{1 / 3}} y_{1}\left(3 k t_{0}^{2 / 3} t^{1 / 3}\right),
$$

where $j_{1}$ and $y_{1}$ are spherical Bessel function of the first kind and that of the second kind, respectively. By using the particular solution and the homogeneous solutions (29), we obtain the general solution of Eq. (27) as

$$
\tilde{y}_{\mathbf{k}}(t)=\frac{S_{\mathbf{k}}\left(t_{0}\right)}{k^{2}}+C_{1}(\mathbf{k}) \tilde{y}_{\mathbf{k}, 1}(t)+C_{2}(\mathbf{k}) \tilde{y}_{\mathbf{k}, 2}(t),
$$

where $C_{1}$ and $C_{2}$ are arbitrary functions. From Eqs. (25) and (30), we obtain the metric perturbation $\tilde{\chi}^{(2)}$ as

$$
\tilde{\chi}^{(2)}(t, \mathbf{x})=r^{2} \int \frac{d^{3} k}{(2 \pi)^{3 / 2}} e^{i \mathbf{k} \cdot \mathbf{x}} \frac{S_{\mathbf{k}}\left(t_{0}\right)}{k^{2}}+(\text { decaying modes }) .
$$

By using Eqs. (21) and (31), we obtain

$$
\tilde{\varsigma}^{(2)}(t, \mathbf{x})=-\frac{3}{5} t^{1 / 3} t_{0}^{2 / 3} \partial_{r} \tilde{\chi}^{(2)}(t, \mathbf{x})+(\text { decaying modes }) .
$$

From Eqs. (31) and (32), we can see that $\tilde{\chi}^{(2)}$ and $\tilde{\varsigma}^{(2)}$ do not vanish: In general, $\tilde{\chi}^{(2)}$ is temporally constant and $\tilde{\varsigma}^{(2)}$ is proportional to $t^{1 / 3}$ at late time, even if we set $\tilde{\varsigma}^{(1)}=\tilde{\chi}^{(1)}=0$, 
or in other words, $\tilde{\varsigma}$ and $\tilde{\chi}$ vanish up to the order $\epsilon$. Thus, exactly speaking, the assumption proposed by $\mathrm{CCF}$, i.e., Eq. (6) is not valid, if we take the order $\kappa \epsilon$ into account in the evolution of metric perturbations. However, it is worthwhile to notice that the effects of $\tilde{\chi}^{(2)}$ and $\tilde{\varsigma}^{(2)}$ to the density perturbation $\tilde{\delta}^{(2)}$ are less dominant than the other effects, because the terms with respect to $\tilde{\chi}^{(2)}$ and $\tilde{\varsigma}^{(2)}$ in the right hand side of Eq. (22) proportional to $t^{-4 / 3}$ whereas the terms with respect to $\tilde{\varphi}^{(1)}$ proportional to $t^{-2 / 3}$. Thus, the assumption proposed by CCF will be a good approximation for sufficiently late time in the study of the density perturbations.

We expect that $\tilde{\chi}^{(2)}$ and $\tilde{\varsigma}^{(2)}$ become important in testing LTB cosmological models from observations of large-scale structures such as weak gravitational lensing, since a photon from a galaxy propagates in the spacetime with these metric perturbations. We leave the analysis of the effects to the observations for a future work.

February, Larena, Clarkson and Pollney (hereafter FLCP) [64] numerically studied the perturbations in the LTB spacetime without any approximations except for the linearization and discretization of the differential equations governing them, and investigated the validity of the assumption proposed by CCF. FLCP showed that if the coupling of $\tilde{\delta}$ and $\tilde{\varphi}$ to $\tilde{\chi}$ and $\tilde{\varsigma}$ is neglected, errors in $\tilde{\delta}$ and $\tilde{\varphi}$ increase with the spatial length scale of perturbations decreasing and grow over time (see fig. 11 in Ref. 64]). In the present case, we see from Eqs. (201) and (22) that the effects of $\tilde{\chi}^{(2)}$ and $\tilde{\varsigma}^{(2)}$ on $\tilde{\varphi}^{(2)}$ and $\tilde{\delta}^{(2)}$ may also increase with the spatial scale of perturbations decreasing, since the dominant terms related to $\tilde{\chi}^{(2)}$ and $\tilde{\varsigma}^{(2)}$ in the equations for $\tilde{\varphi}^{(2)}$ and $\tilde{\delta}^{(2)}$ are their spatial derivatives. Thus, regarding the spatial scale dependence of the errors, our results seem to be consistent with those of FLCP. As for the time dependence of the errors, our results imply that the errors decrease over time, and this consequence contradicts the results obtained by FLCP. We guess that the difference comes from the nonlinear effects of the spherical void; we focused on the situation in which the effects of a void structure are so small that they can be treated perturbatively, i.e., we assumed $\kappa \ll 1$, whereas FLCP assumed a highly nonlinear void structure at the present time (see Eq. (10) in Ref. [64]), i.e., the background LTB spacetime satisfies $\kappa \gg 1$ at the present time.

\section{Acknowledgments}

KN was supported in part by JSPS Grant-in-Aid for Scientifc Research (C) (No. $25400265)$.

[1] P. Bull and T. Clifton, Phys. Rev. D 85, 103512 (2012).

[2] M. N. Celerier, Astron. Astrophys. 353, 63 (2000).

[3] M. N. Celerier, K. Bolejko and A. Krasinski, Astron. Astrophys. 518, A21 (2010). 
[4] T. Clifton, P. G. Ferreira and K. Land, Phys. Rev. Lett. 101, 131302 (2008).

[5] S. P. Goodwin, P. A. Thomas, A. J. Barber, J. Gribbin and L. I. Onuora, arXiv:astro-ph/9906187.

[6] H. Iguchi, T. Nakamura and K. i. Nakao, Prog. Theor. Phys. 108, 809 (2002).

[7] E. W. Kolb and C. R. Lamb, arXiv:0911.3852 [astro-ph.CO].

[8] N. Mustapha, C. Hellaby, G. F. R. Ellis, Mon. Not. Roy. Astron. Soc. 292, 817-830 (1997).

[9] K. Tomita, Astrophys. J. 529, 38 (2000).

[10] K. Tomita, Mon. Not. Roy. Astron. Soc. 326, 287 (2001).

[11] K. Tomita, Prog. Theor. Phys. 106, 929 (2001).

[12] R. A. Vanderveld, E. E. Flanagan and I. Wasserman, Phys. Rev. D 74, 023506 (2006).

[13] C. M. Yoo, T. Kai and K. i. Nakao, Prog. Theor. Phys. 120, 937 (2008).

[14] C. -M. Yoo, Prog. Theor. Phys. 124, 645-665 (2010).

[15] S. Alexander, T. Biswas, A. Notari and D. Vaid, JCAP 0909, 025 (2009).

[16] H. Alnes, M. Amarzguioui and O. Gron, Phys. Rev. D 73, 083519 (2006).

[17] T. Biswas, A. Notari and W. Valkenburg, JCAP 1011, 030 (2010).

[18] K. Bolejko and J. S. B. Wyithe, JCAP 0902, 020 (2009).

[19] C. Clarkson and M. Regis, JCAP 1102, 013 (2011).

[20] J. Garcia-Bellido and T. Haugboelle, JCAP 0804, 003 (2008).

[21] V. Marra and A. Notari, Class. Quant. Grav. 28, 164004 (2011).

[22] V. Marra and M. Paakkonen, JCAP 1012, 021 (2010).

[23] A. Moss, J. P. Zibin and D. Scott, Phys. Rev. D 83, 103515 (2011).

[24] S. Nadathur and S. Sarkar, Phys. Rev. D 83, 063506 (2011).

[25] C. M. Yoo, K. i. Nakao and M. Sasaki, JCAP 1007, 012 (2010).

[26] J. P. Zibin, A. Moss and D. Scott, Phys. Rev. Lett. 101, 251303 (2008).

[27] J. Garcia-Bellido and T. Haugboelle, JCAP 0909, 028 (2009).

[28] M. Zumalacarregui, J. Garcia-Bellido and P. Ruiz-Lapuente, JCAP 1210, 009 (2012).

[29] P. Bull, T. Clifton and P. G. Ferreira, Phys. Rev. D 85, 024002 (2012).

[30] J. Garcia-Bellido and T. Haugboelle, JCAP 0809, 016 (2008).

[31] A. Moss and J. P. Zibin, Class. Quant. Grav. 28, 164005 (2011).

[32] C. M. Yoo, K. i. Nakao and M. Sasaki, JCAP 1010, 011 (2010).

[33] P. Zhang and A. Stebbins, Phys. Rev. Lett. 107, 041301 (2011). 
[34] P. A. R. Ade et al. [Planck Collaboration], Astron. Astrophys. 561, A97 (2014).

[35] M. Adachi and M. Kasai, Prog. Theor. Phys. 127, 145 (2012).

[36] H. Alnes and M. Amarzguioui, Phys. Rev. D 75, 023506 (2007).

[37] K. Bolejko, M. -N. Celerier and A. Krasinski, Class. Quant. Grav. 28, 164002 (2011).

[38] K. Bolejko, PMC Phys. A 2, 1 (2008).

[39] R. R. Caldwell and N. A. Maksimova, Phys. Rev. D 88, no. 10, 103502 (2013).

[40] M. -N. Celerier, arXiv:1203.2814 [astro-ph.CO].

[41] C. Clarkson, Comptes Rendus Physique 13, 682 (2012).

[42] R. de Putter, L. Verde and R. Jimenez, JCAP 1302, 047 (2013).

[43] P. Dunsby, N. Goheer, B. Osano and J. P. Uzan, JCAP 1006, 017 (2010).

[44] K. Enqvist, M. Mattsson and G. Rigopoulos, JCAP 0909, 022 (2009).

[45] K. Enqvist and T. Mattsson, JCAP 0702, 019 (2007).

[46] H. Goto and H. Kodama, Prog. Theor. Phys. 125, 815 (2011).

[47] A. F. Heavens, R. Jimenez and R. Maartens, JCAP 1109, 035 (2011).

[48] M. Quartin and L. Amendola, Phys. Rev. D 81, 043522 (2010).

[49] M. Regis and C. Clarkson, Gen. Rel. Grav. 44, 567 (2012).

[50] A. E. Romano, Phys. Rev. D 82, 123528 (2010).

[51] A. E. Romano, M. Sasaki and A. A. Starobinsky, Eur. Phys. J. C72, 2242 (2012).

[52] A. E. Romano and P. Chen, JCAP 1110, 016 (2011).

[53] M. Tanimoto, Y. Nambu and K. Iwata, arXiv:0906.4857 [astro-ph.CO].

[54] K. Tomita, arXiv:0906.1325 [astro-ph.CO].

[55] K. Yagi, A. Nishizawa and C. -M. Yoo, J. Phys. Conf. Ser. 363, 012056 (2012).

[56] J. P. Zibin, Phys. Rev. D 84, 123508 (2011).

[57] W. Valkenburg, M. Kunz and V. Marra, Phys. Dark Univ. 2, 219 (2013).

[58] W. Valkenburg, V. Marra and C. Clarkson, MNRAS 438, (2014) L6.

[59] U.H. Gerlach and U.K. Sengupta, Phys. Rev. D 19, 2268 (1979).

[60] D. Alonso, J. Garcia-Bellido, T. Haugboelle and A. Knebe, Phys. Dark Univ. 1, 24 (2012).

[61] D. Alonso, J. Garcia-Bellido, T. Haugbolle and J. Vicente, Phys. Rev. D 82, 123530 (2010).

[62] C. Clarkson, T. Clifton and S. February, JCAP 0906, 025 (2009).

[63] S. February, C. Clarkson and R. Maartens, JCAP 1303, 023 (2013).

[64] S. February, J. Larena, C. Clarkson and D. Pollney, Class. Quant. Grav. 31, 175008 (2014). 
[65] J. P. Zibin, Phys. Rev. D 78, 043504 (2008).

[66] E. Bertschinger and A. J. S. Hamilton, Astrophys. J. 435, 1 (1994).

[67] R. Nishikawa, C. -M. Yoo and K. -i. Nakao, Phys. Rev. D 85, 103511 (2012).

[68] R. Nishikawa, C. -M. Yoo and K. -i. Nakao, Phys. Rev. D 88, 123520 (2013).

[69] S.W. Hawking and G.F.R. Ellis, "The large scale structure of space-time," (Cambridge Universe Press 1973). 